\title{
Perceived angle of incidence and achromatic surface color ${ }^{1,2}$
}

\author{
HOWARD R. FLOCK ${ }^{3}$ AND EDMUND FREEDBERG \\ YORK UNIVERSITY
}

\begin{abstract}
Theory and evidence proposing illumination, and therefore necessarily angle of incidence of light, as a perceptual cue for lightness judgments are described. In two experiments, angle of incidence was varied by having $46 \mathrm{Ss}$ view an upright trapezoid that monocularly appeared flat and binocularly, upright. In the first experiment, numbers of cues to the direction of a fixed source above the trapezoid were varied and combined. In a second experiment, the background for an illumination gradient was varied. Angle of incidence and cues to the location of the source did predict the trends in lightness judgments but not the magnitude of the effects. An alternative explanation to cue theory is proposed.
\end{abstract}

In providing an account for the constancy of perceived achromatic surface color, a body of opinion exists that proposes that one of its determinants is the perception (or registration) of the illumination of a surface. The theoretical background (Helmholtz, 1925; Katz, 1935), upon which this body of opinion seems to be based, failed to make explicit the implications of the theory as they applied to the perception of achromatic surface color, although attempts to do this have been made (Hochberg \& Beck, 1954; Katz, 1935; Woodworth, 1938, Chap. 24; Woodworth \& Schlosberg, 1954, Chap. 15). If a test is to be made of the cue value of the illumination in perceptions of achromatic surface color, what is required first is an explicit account of the spatial variables on which any perception of illumination must depend and of how these are related to the problems of the constancy of perceived achromatic surface color. (The terms, "albedo perception," "lightness perception," "reflectance perception," and even "the brightness of surfaces," have been used interchangeably with the term, "achromatic surface color.")

The arguments for this inferential cue theory (or empiricist "inferential" theory) have depended as much on its reasonableness as on empirical evidence for it. Consider first its reasonableness. The luminance of a surface is determined by the illuminance (illumination) and by the characteristics of the surface (reflectance). Therefore, it would be impossible to demonstrate the constancy of achromatic surface colors were luminance of a surface to be the sole stimulus correlate for the perception of its achromatic color. It is generally claimed, however, that Os do demonstrate considerable constancy in their perceptions of achromatic colors despite the changes in illuminance. Ergo, it must follow that the illuminance as well as the luminance are registered by the perceiver.

This version of inferential cue theory, often called albedo theory, corresponds to that given by Woodworth (1938) and Woodworth and Schlosberg (1954). It could be expressed as follows:

$$
R=\frac{L}{E},
$$

where $R$ is the reflectance of a surface (or apparent surface color), $L$ is the luminance of a surface, and $E$ is the illuminance of a surface.

Woodworth (1938) and Katz (1935) understood that perception of the illumination implied perception of the angle of incidence of the light on a surface. It might be added that it also implied perception of the distance between a source and a surface and the perception of the intensity of the source. The term for illuminance, E, should be expanded, therefore, so that Eq. 1 becomes:

$$
R=I \frac{d^{2}}{I \cos \theta},
$$

where $I$ is the luminous intensity of a point source, $\theta$ is the angle of incidence of light ${ }^{4}$; $d$ is the distance between surface and source, and $R$ and $L$ are as defined above.

In albedo theory, it is assumed that the o solves for the surface reflectance, $R$, by giving values to the terms in Eq. 2 . If the values are appropriate, then correct perceptions of surface lightness occur. Whereas luminance is given by the light to the eye,$^{5}$ it is less clear what the stimulus correlates are for the remaining terms. Nevertheless, one might assume that the $O$ uses cue information to yield estimates for these terms. The cue basis for estimating the intensity of a source could be the luminance of attached and cast shadows, luminance gradients on backgrounds and surfaces, the overall average luminance of a scene, the highest luminance in a scene, and perhaps even the visible presence of a source. The direction of the source could be estimated from the shapes and locations of shadows, highlights, and background gradients. The orientation of a surface could be derived from the relevant visual and nonvisual cues, as could the distance between a source and a particular surface.

Were it necessary to amass all of this information, it might seem that this theory was not too reasonable. The task of an $\mathrm{O}$, however, might be simplified, as in the following paradigm

$$
\frac{R_{1}}{R_{2}}=\frac{d_{1}^{2} L_{1} I_{2} \cos \theta_{2}}{d_{2}^{2} L_{2} I_{1} \cos \theta_{1}},
$$

where the subscripts identify the variables associated with two different surfaces.

Equation 3 is the theoretical paradigm for the discrimination of a change in apparent achromatic surface color (reflectance). The advantage of Eq. 3 over Eq. 2 is in the substitution of relative for absolute values. For example, were an $\mathrm{O}$ asked to judge whether or not two surfaces presented in sunlight had the same grayness, under Eq. 2 he would be required to get a measure of the intensity of the sun and its distance! Under Eq. 3, he would be required to note only that both surfaces were in sunlight and that, therefore, $\mathrm{d}_{1}=\mathrm{d}_{2}$ and $\mathrm{I}_{1}=\mathrm{I}_{2}$. With those terms cancelled, his judgment of the two achromatic colors would presumably be determined by cues specifying the angles of incidence of the light and by the two luminances. The theory as embodied in Eq. 3 is more reasonable, indeed. But it has one important disadvantage. Although it predicts change, amount of change, and direction of change, it does not predict what particular achromatic color a surface will have as does Eq. 2.

Katz (1935) believed that this was not an insurmountable difficulty. If a specific level of daylight illumination were considered "normal" for a particular $O$, if that level specified an arbitrary value for the ratio, $\mathrm{d}^{2} / \mathrm{I}$, and if that arbitrary value represented an internalized standard for the $\mathrm{O}$, then, for any observed illuminance, $E$, the $O$ would estimate the degree of departure from his internalized standard rather than $\mathrm{d}^{2} / \mathrm{I}$. In addition, he would estimate the angle of incidence of the light.

Equations 2 and 3 with Katz's internalized standard define albedo theory, 
which, properly, is a subset of inferential cue theory. Equation 3, otherwise, defines a second subset, to be called relative albedo theory and to be tested in this paper. As an example of data that may be cited as defining relative albedo theory, consider an experiment of Hochberg and Beck (1954).

Hochberg and Beck (1954) used an ingenious arrangement to vary the perceived spatial orientation of a surface to a light source without changing any of the other variables given in Eq. 3. This was done by the simple device of having their Ss observe an upright trapezoid with two eyes, thereby reporting the figure as upright, and with one eye, thereby reporting the figure as flat on the table even though it was physically upright. With this procedure, the luminance of the trapezoid, the location and intensity of the source, the distance between source and surface, and the actual reflectance of the surface remained unchanged over the two viewing conditions.

Although the light source was not visible, cues to its location were supplied. Piles of blocks were distributed over the scene. The shapes and locations of the attached and cast shadows would indicate the direction of the source. With cues to the location of the source and with the perceived orientation of the surface, the conditions would exist that define angle of incidence. Hence, angle of incidence could be assumed to have been a variable in the experiment.

The shadows in the scene could have served a second function. Their unchanging luminance would have indicated that the intensity and distance of the source was constant for monocular and binocular viewing. As a result, the only variable in Eq. 3 that was not constant over the experiment was angle of incidence. Thus, all of the terms in Eq. 3 would drop out and the theoretical paradigm for Hochberg and Beck's (1954) experiment could be expressed as:

$$
\mathrm{R}_{\mathrm{m}} / \mathrm{R}_{\mathrm{b}}=\cos \theta_{\mathrm{b}} / \cos \theta_{\mathrm{m}},
$$

where $\mathrm{m}$ and $\mathrm{b}$ refer to monocular and binocular conditions.

For the experiment under consideration, $R_{m}$ and $R_{b}$ might be more usefully read as a monocular response, $R_{m}$, and a binocular response, $R_{b}$, where $R_{b}$ is given some arbitrary value like .5 . If the two angles of incidence were the same, then $R_{m}=.5$, and the surface would appear the same under both binocular and monocular viewing. Were the angle to be less under monocular viewing (and therefore its cosine increased), the $R_{m}$ would be less than .5 and the surface would appear darker for monocular viewing.
Correspondingly, were the angle to be greater under monocular viewing, the surface would appear lighter under monocular viewing.

A general rule can be stated, based on Eqs. 3 and 4: As the angle of incidence of light on a surface is decreased, the surface will appear darker if $\mathrm{L}, \mathrm{I}$, and $\mathrm{d}$ are constant.

When Hochberg and Beck (1954) placed the source above and slightly in front of the upright trapezoid, the apparent angle of incidence for the surface when upright was $85.7 \mathrm{deg}$; for the surface when flat, it was 4.3 deg. ${ }^{6}$ Hence, according to the rule above, the surface should have appeared darker when apparently flat. When the light source was placed in front of the trapezoid and just below the $O$, then binocular viewing gave the smaller angle of incidence. Thus, the surface should appear darker for binocular viewing. Finally, with the source placed laterally to the side of the target, the angle of incidence would be the same for monocular and binocular viewing. There would then be no change in surface lightness.

Under these various conditions, Hochberg and Beck's (1954) Ss invariably reported the surface to be darker when the conditions specified a decreased angle of incidence. The investigators concluded that "the empiricist or 'inferential' position, disconcertingly enough, seems well able to explain the findings ...."

In discussing their experiments, Hochberg and Beck stated that the blocks were of considerable importance as cues: They reported that without them, as observed in preliminary experiments, there was little or no success in achieving the different responses between monocular and binocular viewing that were described above. Presumably, when the visible shadows that were the cues to the direction of the light source were removed, the illumination was perceived as being diffuse. Under those conditions, the ratio of cosines would be expected to be equal to 1.0.

Beck (1965, Study II) replicated Hochberg and Beck (1954) with visible cues to the direction of the source and with the source objectively above the target. Under two of his conditions (Positions 1 and 2), the light source may have been objectively behind the trapezoid. Were that the case, then under binocular viewing, the angle of incidence was indeterminate. Nevertheless, his results were as if the angle of incidence had been smaller for monocular viewing. Under the remaining conditions (Positions 3 and 4), the presumed angle of incidence was either the same or only slightly different from binocular to monocular viewing.
Correspondingly, the judgments of achromatic color tended to be the same.

Beck (1965) measured the magnitude of the effect in Munsell units, finding it to be approximately half of a Munsell step for Positions 1 and 2. Nevertheless, because the reflectance characteristics of Munsell paper are meanıngful only for a standard illuminant and for an objective angle of incidence of $45 \mathrm{deg}$, the median Munsell values he gave cannot be translated into any general expression of magnitude.

In his conclusions, Beck (1965) expressed doubt that the perceived illumination would always affect perceptions of achromatic color. It would depend, he thought, on other prevaling conditions. This could be interpreted to mean that as the cues to the location of the source are increased, the more probable that the perceived angle of incidence would contribute to the resulting perception of surface lightness. This conclusion is to be contrasted to his earlier ones (Beck, 1959, 1961), that perceptions of the illumination might covary with perceptions of surface color under some conditions but were not their determinants.

It is the purpose of the study reported here to take another look at angle of incidence as a cue in the perception of surface color, to see if angle of incidence increasingly determines the perception of a surface color as the cues to the location of the source are varied, to measure the magnitude of its effects for the given cue conditions, and to compare the magnitude of the effects with those specified by relative albedo theory and Eq. 4 .

\section{METHOD}

\section{Subjects}

Twenty-four women, nursing affiliates at Ontario Hospital, Whitby, aged 20 to 22 , participated. No $S$ was allowed to use glasses and no $S$ was accepted for the experiment who needed to use glasses for driving an automobile or for reading a book. All Ss were naive with respect to the experiment.

\section{Apparatus}

An upright trapezoid, with its base standing on a $10 \times 4 \mathrm{ft}$ black velvet horizontal surface, was covered with 5.5. neutral (N) Munsell paper. The upper and lower bases and the height were $5.5,6$, and 3.6 in. A pair of 4-in. wooden cubes was placed $6 \mathrm{in}$. in front of the trapezoid and 4 in. to either side of the midline. A second pair of cubes was placed 24 in. in front and 3 in. to either side of the midline. In order to change the background, a sheet of white Craftint paper, $18 \times 24$ in., corresponding to a Munsell N 8, could be placed on the black velvet surface. These arrangements are shown in Fig. 1 a. 


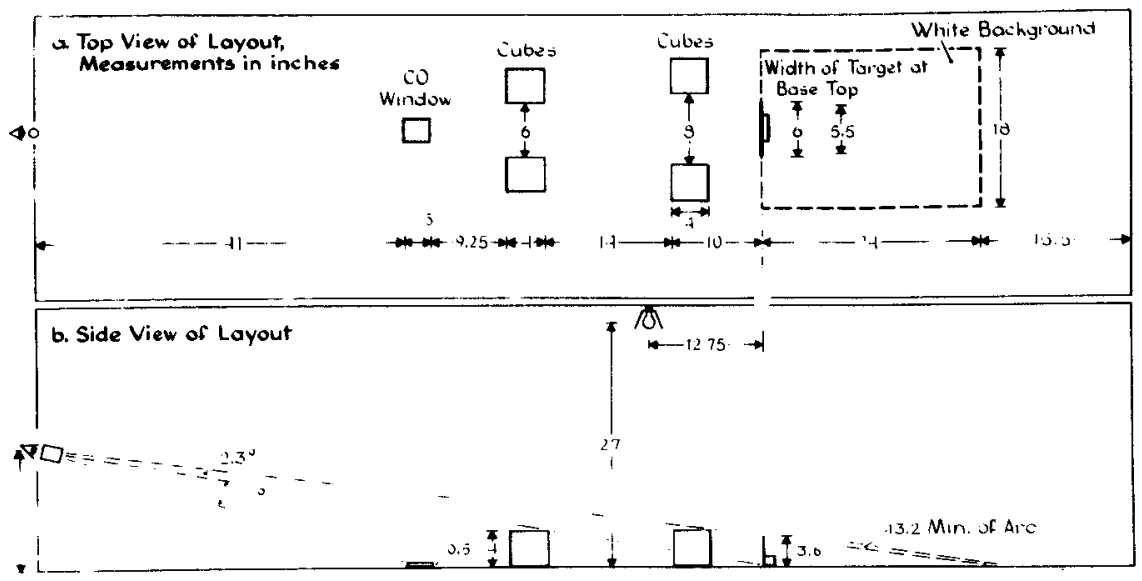

A 2.5-in. square window was introduced into the black velvet, $37.25 \mathrm{in}$. in front of the trapezoid. A series of 17 Munsell patches from $\mathrm{N} 1.5$ to $\mathrm{N} 9.5$ could be presented individually in this window in an ascending or descending order. A hooded $100-\mathrm{W}$ household tungsten lamp was placed $27 \mathrm{in}$. above and $12.75 \mathrm{in}$. in front of the trapezoid, measured from the midpoint of the lower base. Two apertures, each with an inner diameter of $1.4 \mathrm{in}$. and $2 \mathrm{in.} \mathrm{long,}$ were located $80.75 \mathrm{in}$. in front of and 14.25 in. above the midpoint of the lower base of the trapezoid. The eyepieces were rotated downward $20 \mathrm{deg}$ and were converged inward $13 \mathrm{deg}$. They also could be adjusted for differences in interocular distances.

A black disk could be introduced into either eyepiece to occlude the scene from that eye. With either eye there was an unobstructed view of the trapezoid, of the window with the comparison ( $\mathrm{CO}$ ) series, of the four cubes, and of part of a black velvet wall at a distance of $10 \mathrm{ft}$. The lamp could not be seen.

It should be noted in Fig. $1 \mathrm{~b}$ that, when the white background was in place, only a strip of it, $13.2 \mathrm{~min}$ of arc in the vertical meridian, could be seen over the top of the trapezoid. The angular width of the trapezoid along its lower base was $4.2 \mathrm{deg}$, whereas the angular width of the white background at the same location was $12.5 \mathrm{deg}$. When the cubes were introduced, they occluded a large part of the white background, leaving only a section of it, $2.2 \mathrm{deg}$ in angular width, adjacent to either side of the lower base of the trapezoid. With upward angulation of the eye, increasingly more of the white background was visible. The angular height of the target was $2.3 \mathrm{deg}$. The top faces of the cubes nearer the trapezoid were $3 \mathrm{deg}$ in angular

Fig. 2. Distribution of luminances (foot-lamberts) over scene, target, and CO series. width along the edge near the trapezoid and were $.45 \mathrm{deg}$ in angular depth. The front surfaces of these cubes were $3.6 \mathrm{deg}$ in angular height. The top faces of the pair of cubes farther from the trapezoid were in
Fig. 1. (a) Top view of layout, measurements in inches. (b) Side view of layout.

angular dimensions, $4 \mathrm{deg}$ in width, $.78 \mathrm{deg}$ in depth, and the front faces were $4.95 \mathrm{deg}$ in height.

Figure 2 gives some measurements in foot-lamberts of the distribution of luminances over various parts of the scene. The Xs mark the locations of measurements of parts of the white background and the faces of the cubes. The solid dots indicate the locations of measurements of the black background. The trapezoidal inset gives a set of measurements of the face of the upright trapezoid; the other inset gives a set of measurements of the $\mathrm{CO}$ series. It should be noted that the ratio of luminances between the trapezoid and its immediate black background varied from approximately $35: 1$ in the foreground to

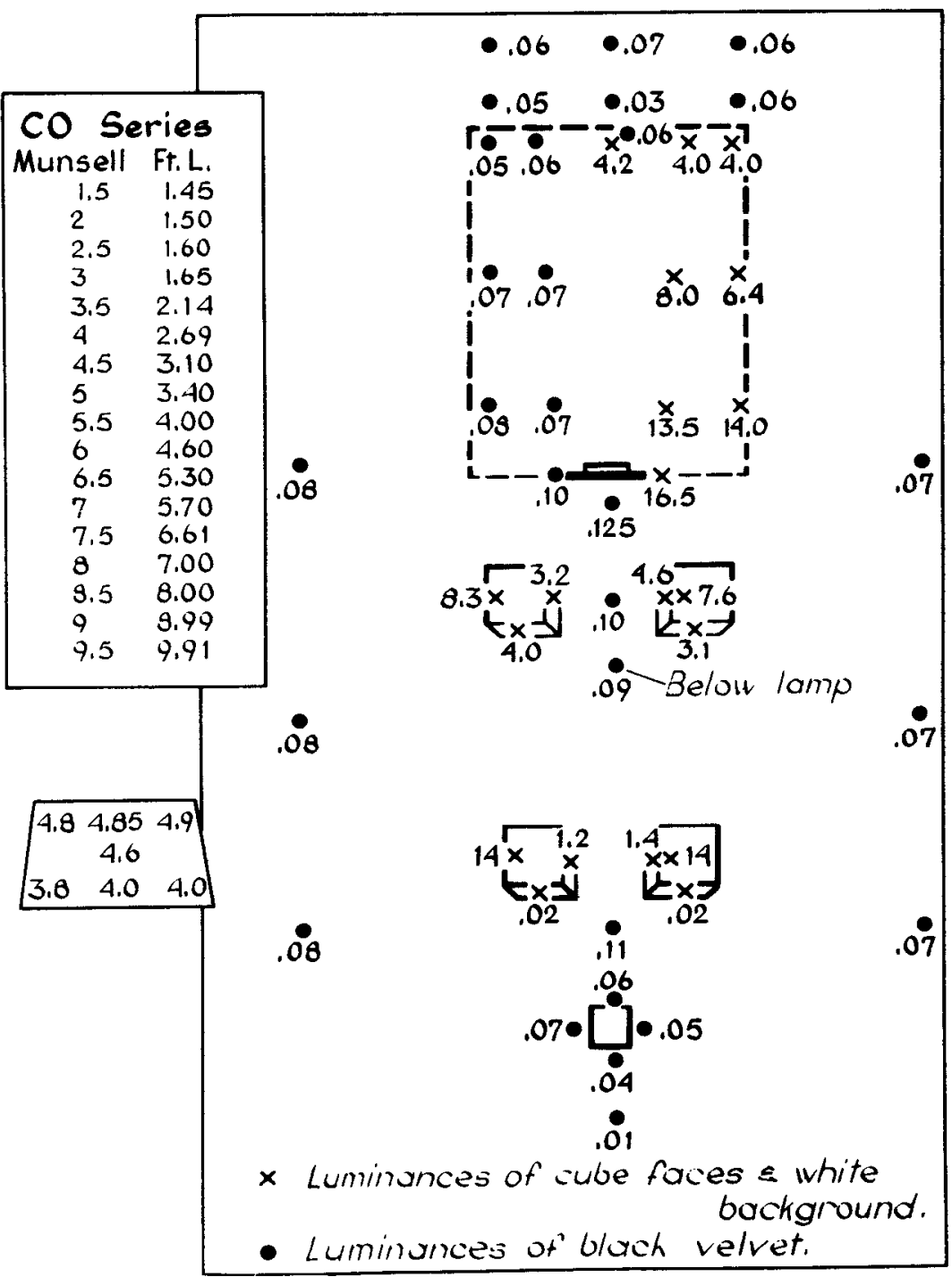


Fig. 3. Mean lightness judgments in log luminances for binocular and monocular viewing with and without cubes on a black and white background.

$75: 1$ in the background, whereas between the trapezoid and the white background, the variation was approximately from 1:4 to $1: 1$. Finally, it should be noted that there was a gradient of luminances on the black background from $.125 \mathrm{ft}-\mathrm{L}$ in front of the trapezoid to $.03 \mathrm{ft}-\mathrm{L}$ in the background and to $.04 \mathrm{ft}-\mathrm{L}$ and $.01 \mathrm{ft}-\mathrm{L}$ in the near foreground. For the white background, the gradient was from 16.5 to $4 \mathrm{ft}-\mathrm{L}$ adjacent to the target.

\section{Procedure}

Each S was instructed to choose the Munsell $\mathrm{CO}$ that appeared to be the same gray as the target (trapezoid). The $\mathrm{CO}$ series was presented in either an ascending or descending order, but $S$ could instruct the $E$ to reverse the direction of the series until he was satisfied with his choice. The method, therefore, was a modified method of adjustment. The $S$ was allowed to look back and forth from the target to the $\mathrm{CO}$ and to take as much time as he wished. Between each trial, a shutter occluded the display and the $S$ dark-adapted in a dimly illuminated space for approximately $30 \mathrm{sec}$. The $\mathrm{S}$ was dark-adapted for $10 \mathrm{~min}$ at the start of the experiment.

At the beginning of the experiment, the Ss inspected the display monocularly and were asked to describe the spatial position of the target. If they reported it to be flat, the experiment continued. If they failed to report it to be flat, they were given a second trial. If they did not then report it to be flat, they were dismissed from the experiment, which was the case with several Ss. Before each of the first 10 trials, Ss were required to report the position of the target, whether flat, slanted, or vertical. All Ss reported it flat or slanted under monocular viewing and vertical under binocular viewing.

Each $S$ made one judgment of the grayness of the target for each of 16 treatment conditions: monocular and binocular viewing by cubes and no cubes by black and white background by ascending and descending presentations of the $\mathrm{CO}$ series. The 16 treatments were presented in a different random order to each $S$, but the left and right eyes were used equally often under monocular viewing.

\section{RESULTS AND DISCUSSION}

\section{Contrast Effects}

The data $^{7}$ were converted to $\log$ foot-lamberts, and the ascending and descending scores for a $S$ were averaged, giving eight mean log judgments for each of the 24 Ss. In Fig. 3, the means of these

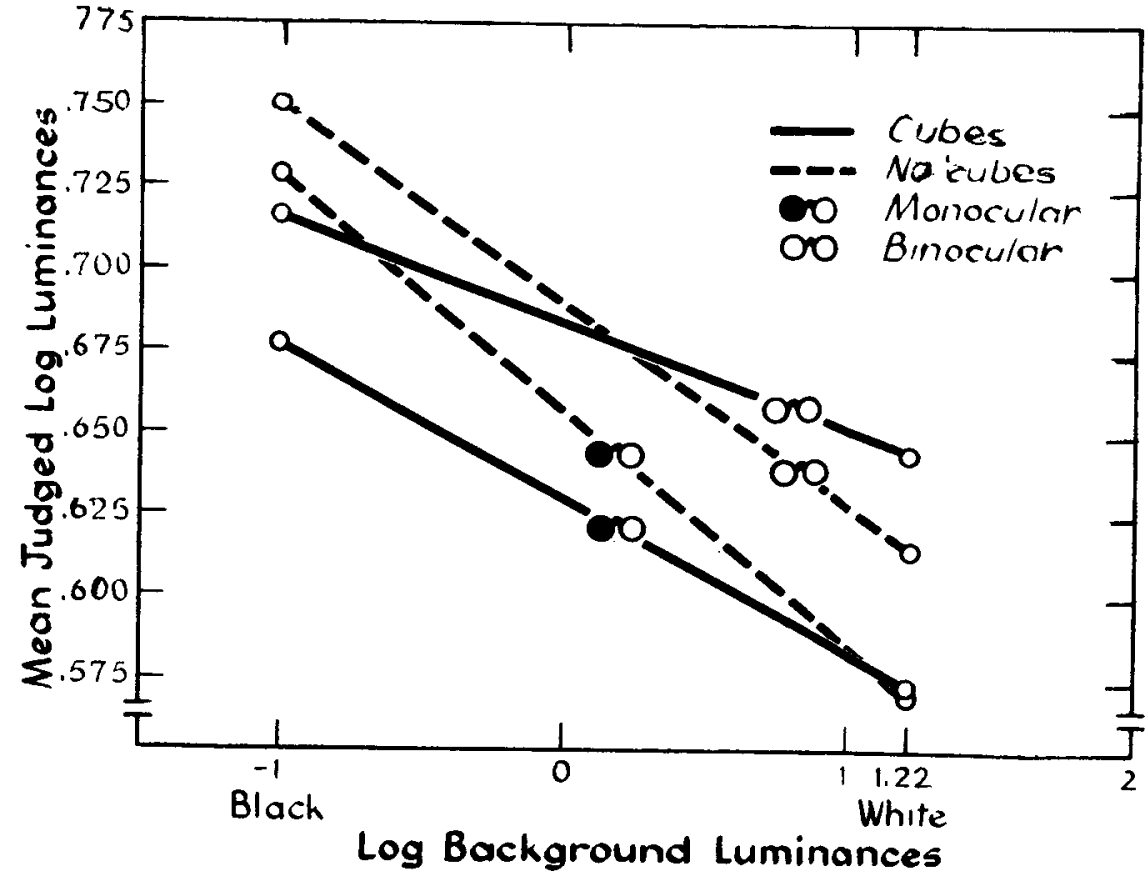

have been plotted against the black $(-1.0 \log f t-L)$ and white $(1.22 \log f t-L)$ background luminances. An analysis of variance yielded three significant effects: background $(\mathrm{F}=38.9, \mathrm{df}=1 / 23, \mathrm{p}<.01)$, cubes by background $(F=17.6, \mathrm{df}=1 / 23$, $p<.01)$ and viewing $(F=14.4, d f=1 / 23$, $p<.01)$. These effects are all evident in Fig. 3.

The significant background effect is represented by the negative slopes of all four lines, indicating that the mean responses were of a darker gray target when the background was white rather than black velvet. This would be expected because of the higher luminances surrounding the target when the white background was in place (Diamond, 1953). From Fig. 2 it can be seen that when the white background replaced the black velvet background, the surround luminances adjacent to the target changed by a factor of approximately $165: 1$ or by $2.2 \mathrm{log}$ units.

The significant cubes by background effect is represented in Fig. 3 by the flatter negative slopes of the two lines that describe the responses when cubes had been introduced, and by the steeper negative slopes of the two lines that describe the responses when the cubes had been removed. This, too, would have been expected to follow from the distribution of surround luminances. When the cubes were introduced, they occluded large angular sections of the immediate black velvet surround, replacing those regions of lower reflectance with regions (the faces of the cubes) of higher reflectance, a change in luminance ratio of 100:1 (see Fig. 2). Moreover, with the angular separation between the trapezoid and the nearest face of a cube being $2.2 \mathrm{deg}$, the separation was within the range in which induction effects might be expected to take place (Leibowitz, Mote, \& Thurlow, 1953). This explains the displacement downward of the two data points for cubes on a black background.

There should be a corresponding displacement upward of the two data points for cubes on a white background, and for the same reasons. The cubes also occluded a considerable amount of the white background, thereby replacing a brighter with a less bright surround. The result should have been an upward displacement of the two data points. The data point for binocular viewing with white background and cubes was displaced upward, but the data point for monocular viewing with white background and cubes was not. We have no explanation for that discrepancy: it may represent a chance variation, it may be the result of a somewhat different angular distribution of luminances that occurred for a monocular vs binocular viewing, or it may be confounded with the viewing effect described below.

The viewing effect. In the results above, the evidence for the operation of contrast mechanisms has been emphasized. It is less clear, however, how these mechanisms could explain the significant viewing (monocular vs binocular) effect. When viewed monocularly, the target elicited darker Munsell choices than when viewed 
Table 1

Mean Judged Log Luminances and Differences Between Monocular and Binocular Viewing

\begin{tabular}{lcccccccc} 
& \multicolumn{2}{c}{ Black Background } & & & \multicolumn{3}{c}{ White Background } \\
\cline { 2 - 3 } \cline { 7 - 9 } & Monocular & Binocular & $D$ & & & Monocular & Binocular & $D$ \\
\hline Cubes & .677 & .716 & .039 & & .573 & .644 & .071 \\
No Cubes & .729 & .750 & .021 & & .570 & .614 & .044 \\
\hline
\end{tabular}

binocularly. This effect occurred on a white background, on a black background, with cubes, and without cubes, as is clearly shown in Fig. 3.

Effect of cues. The rule for this experiment developed from inferential cue theory, and Eq. 4 stated that Ss would report a surface to be darker when its angle of incidence decreased. That prediction was confirmed in this experiment by the significant viewing effect. Nevertheless, the prediction depends on the presence of detectable cues to the direction of the light source. A hypothesis stated in the introduction was that the magnitude of the viewing effect might depend on the number and type of these cues.

In this experiment, there were two different classes of cues to the location of the source: attached shadows and highlights on the cubes, and background gradients. Presumably, the background gradients were less detectable on the black background, where the change in luminances adjacent to the target was from .10 to $.03 \mathrm{ft}-\mathrm{L}$ (see Fig. 2), and were more detectable on the white background, where the change was from 16.5 to $4.0 \mathrm{ft}-\mathrm{L}$. The two classes of cues were also combined, as in presenting cubes and white background together.

The trends in Table 1 (and Fig 3) support the argument that, as the number of detectable cues is increased, the magnitude of the viewing effect is increased. The difference (D) in monocular and binocular viewing was least $(D=.021)$ when background gradients were presumably less detectable, was intermediate when background gradients were more detectable $(D=.044)$ or when

there were attached shadows $(D=.039)$, and was greatest when the more detectable background gradients and attached shadows were combined $(D=.071)$. This interpretation was supported also in Table 2 by the frequency with which the target was monocularly seen darker $(+)$ or lighter (-) for the ascending and descending series and for the four levels of cues. The proportions (P) changed from .56 to .88 . Nevertheless, this account was not supported by the analysis of variance ( $F=1.3$, df $=1 / 23$, for background by viewing; $F=1.5, \mathrm{df}=1 / 23$, for blocks by viewing; $F=.10, \mathrm{df}=1 / 23$, for background by blocks by viewing).

The same trends and the same statistical nonsignificance occurred in a second experiment. Twenty-two Ss judged the lightness of the trapezoid presented on a white, gray, and black background. All other conditions were the same except that there were no cubes in the scene. As the background was changed from black through gray to white, presurnably the background gradient specifying the location of the source became increasingly detectable and the viewing effect would correspondingly be expected to increase. The difference between monocular and binocular judgments was .03, .04, and $.07 \log \mathrm{ft}-\mathrm{L}$ for the black, gray, and white backgrounds, with the monocular judgments darker than the binocular judgments, as before. This occurrence of the trend on a second occasion would suggest that the trend was probably reliable. (The independent probability of each trend being $1 / 6$, the probability of both was $1 / 36$, or $\mathrm{p}<.05$ ).

There might be an alternative to

Table 2

Frequency of Monocularly Darker $(+)$ and Lighter $(-)$ Lightness Judgments for Four Levels of Cues

\begin{tabular}{|c|c|c|c|c|c|c|c|c|}
\hline \multirow{2}{*}{-} & \multicolumn{2}{|c|}{$\begin{array}{c}\text { Gradients } \\
\text { (Black } \\
\text { Background) }\end{array}$} & \multicolumn{2}{|c|}{$\begin{array}{c}\text { Gradients } \\
\text { (White } \\
\text { Background) }\end{array}$} & \multicolumn{2}{|c|}{$\begin{array}{l}\text { Attached Shadows } \\
\text { (Cubes and } \\
\text { Black Background) }\end{array}$} & \multicolumn{2}{|c|}{$\begin{array}{l}\text { Combined Cues } \\
\text { (Cubes and } \\
\text { White Background) }\end{array}$} \\
\hline & Asc. & Desc. & Asc. & Desc. & Asc. & Desc. & Asc. & Desc. \\
\hline+ & 11 & 12 & 11 & 12 & 11 & 11 & 15 & 15 \\
\hline- & 9 & 9 & 7 & 6 & 6 & 6 & 2 & 2 \\
\hline Ties & 4 & 3 & 6 & 6 & 7 & 7 & 7 & 7 \\
\hline$P(+)$ & \multicolumn{2}{|c|}{.56} & \multicolumn{2}{|c|}{.64} & \multicolumn{2}{|c|}{.65} & \multicolumn{2}{|c|}{.88} \\
\hline
\end{tabular}

inferential cue theory as an explanation for this trend. An analogy between this experimental situation and the Wertheimer-Benussi gray ring on a black-white bipartite field might be relevant. When the Wertheimer-Benussi ring is unitary, it tends to appear in front of its background and tends to appear homogeneously gray. When the ring is divided, the two half-rings appear embedded in their respective black or white backgrounds. With embeddedness, contrast occurs, the half-ring surrounded by white appearing darker. Since monocular viewing embedded the trapezoidal target in the background, increasing background luminance might increasingly darken the target under monocular viewing. This analogy would not explain, however, why the black velvet background also gave darker monocular judgments, unless increased assimilation was argued to occur under these conditions. Nor would the analogy explain why there was no difference between monocular and binocular judgments when the source was placed to the side of the target by Hochberg and Beck (1954).

Magnitude of the effect. Relative albedo theory, as embodied in Eq. 4, specifies how large the viewing effect should be. In the first experiment, if the target was seen flat when viewed monocularly, the angle of incidence would have been $25.3 \mathrm{deg}$. For the binocularly upright target, it would have been $64.7 \mathrm{deg}$. (These angles can be calculated from the measurements in Fig. 1.) The ratio of cosines for the two angles would then be $2.11: 1$. Converted to a $\log$, this ratio means that there should be a shift of $.32 \log \mathrm{ft}-\mathrm{L}$ from binocular to monocular viewing. In fact, the largest shift in apparent mean log luminance was, as given in Table $1, .071$, and the smallest shift was .021 . These observed values of .021 and .071 were equivalent to a change in orientation of the target (and in this case to a corresponding change in angle of incidence) of 1.4 and $4.9 \mathrm{deg}^{8}$ instead of the presumed change of $90 \mathrm{deg}$ from the upright to the horizontal.

In view of the Ss' reports that the target appeared slanted or flat over the first 10 judgments, and in view of the empirical evidence for the perceived slant of upright trapezoids under conditions not dissimilar to these (Dunn, 1966; Flock, 1965, 1967; Smith, 1967), it doesn't seem reasonable to assume that the monocular target slanted only 1.4 to $4.9 \mathrm{deg}$ on the average. Moreover, even if the presumed change in 
slant was reduced from $90 \mathrm{deg}$ to, for example, $39 \mathrm{deg}$, the expected ratio of cosines would still have been $2.11: 1$. This is the case in the present situation because a 90-deg change in slant of the surface takes the surface through the angle in which angle of incidence would be 0 deg to an angle of opposite sign.

In summary, it would seem that the magnitude of the difference between monocular and binocular judgments was too small to imply that the change in perceived angle of incidence was the sole determinant of change in achromatic surface color. The trends in the data could imply, however, that the lightness judgments depended on angle of incidence as a perceptual cue as well as on the detectability and number of cues to the location of the source. The trends, therefore, might be said to give some support to relative albedo theory and to the more general inferential cue theory.

An alternative explanation to inferential cue theory was also proposed: First, it was shown that the situation was not wholly unique and the judgments, except for the viewing effect, did fluctuate in accordance with expectations based on simultaneous contrast. Second, although the contrast literature could not explain the differences and trends in the differences between monocular and binocular viewing, those differences might have been instances of the Wertheimer-Benussi effect, in which embeddedness would be expected to increase contrast and perhaps assimilation as well.

\section{REFERENCES}

BECK, J. Stimulus correlates for the judged illumination of a surface. Journal of Experimental Psychology, 1959, 58, 267-274.

BECK, J. Judgments of surface illumination and lightness. Journal of Experimental Psychology, $1961,61,368-375$.

BECK, J. Apparent spatial position and the perception of lightness. Journal of Experimental Psychology, 1965, 69, 170-179.

DIAMOND, A. L. Foveal simultaneous brightness contrast as a function of inducing-field luminance. Journal of Experimental Psychology, 1953, 45, 304-314.

DUNN, B. E. Perceived slant as a function of direction of regard. Psychonomic Science, 1966, 4, 297-298.

FLOCK, H. R. Optical texture and linear perspective as stimuli for slant perception. Psychological Review, 1965, 72, 505-514.

FLOCK, H. R. Slant judgments of single rectangles at a slant. Psychonomic Science, $1967,7,57-58$

HELMHOLTZ, H. (Transl. by J. C. P. Southall) Physiological optics. Rochester: Optical Society of America, 1925.

HOCHBERG, J. E., \& BECK, J. Apparent spatial arrangement and perceived brightness. Journal of Experimental Psychology, 1954, 47, 263-266.

KATZ, D. (Transl. by R. B. MacLeod) The world of colour. London: Kegan Paul, 1935.

LEIBOWITZ, H., MOTE, F. A., \& THURLOW, W. Simultaneous contrast as a function of separation between test and inducing fields. Journal of Experimental Psychology, 1953, 46, 453-507.

SMITH, A. H. Perceived slant as a function of stimulus contour and vertical dimension. Perceptual \& Motor Skills, 1967, 24, 167-173. WOODWORTH, R. S. Experimental psychology. New York: Holt, 1938.

WOODWORTH, R. S., \& SCHLOSBERG, H. Experimental psychology. New York: Holt, 1954.

\section{NOTES}

1. A shortened version of this paper was presented at the 1967 meetings of the Eastern Psychological Association in Boston, Massachusetts.

2. This research was supported by the National Research Council of Canada, Grant No. APA-143.

3. Address: Department of Psychology, York University, 4700 Keele Street, Downsview, Toronto, Canada.

4. Measured as the angle formed by the axis normal to a point on the surface and a line connecting that point and the light source.

5. To be more complete, this account of luminance as registered by the visual system should include a term for a variable pupil. Otherwise, a fixed pupil must be assumed.

6. Hochberg, in a personal communication, indicated that the trapezoid was $10 \mathrm{~cm}$ in height, the overhead lamp was $5 \mathrm{~cm}$ in front of the surface, and the front lamp was $5 \mathrm{~cm}$ above the table. The other measurements relevant to a calculation of angle of incidence were given in Fig. 1 of Hochberg and Beck (1954).

7. The tables of raw data may be obtained free of charge from the editorial office of this Journal, Document No. P757.

8. These values were calculated by solving Eq. 4 for $\theta_{2}$, where $R_{1} / R_{2}=$ antilog .021 or antilog .071, and $\theta_{1}=64.7 \mathrm{deg}$, the angle of incidence for the binocularly upright trapezoid, and then by subtracting $\theta_{2}$ from $\theta_{1}$.

(Accepted for publication August 19, 1969.) 